\title{
Zeta: a Resolution Modeling System
}

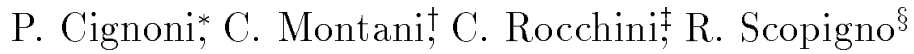 \\ Istituto per l'Elaborazione dell'Informazione - Consiglio Nazionale delle Ricerche \\ Via S. Maria, 46 - 56126 Pisa ITALY \\ *Email: cignoni@iei.pi.cnr.it \\ †Email: montani@iei.pi.cnr.it \\ †Email: rocchini@galileo.iei.pi.cnr.it \\ ${ }^{\S}$ Email: r.scopigno@cnuce.cnr.it
}

May 20, 1998

\begin{abstract}
Very large graphics models are common in a number of applications, and many different simplification methods have been recently developed. Some of them support the construction of multiresolution representations of the input meshes. On the basis of these innovative techniques, we foresee a modeling framework based on three separate stages (shape modeling, multiresolution encoding and resolution modeling), and propose a new approach to the last stage, resolution modeling, which is highly general, user-driven and not strictly tied to a particular simplification method.

The approach proposed is based on a multiresolution representation scheme for triangulated, 2manifold meshes, the Hypertriangulation Model (HyT). This scheme allows to selectively "walk" along the multiresolution surface, moving between adjacent faces efficiently. A prototypal resolution modeling system, Zeta, has been implemented to allow interactive modeling of surface details and evaluated on several practical models. It supports: efficient extraction of fixed resolution representations; unified management of selective refinement and selective simplification; easy composition of the selective refinement/simplification actions, with no cracks in the variable resolution mesh produced; multiresolution editing; interactive response times.
\end{abstract}

CR Descriptors: I.3.5 [Computer Graphics]: Computational Geometry and Object Modeling - Curve, surface, solid and object representation; I.3.6 [Computer Graphics]: Methodology and Techniques.

Additional Keywords: surface modeling, mesh simplification, multiresolution, selective refinements, resolution modeling.

\section{Contact person:}

Roberto Scopigno, I.E.I.- C.N.R., Via S. Maria 46, 56126 PISA (Italy)

Phone: +39 50593304 FAX: +39 50904052

EMail: r.scopigno@cnuce.cnr.it 


\section{Introduction}

Multiresolution representation is a very hot topic, due to the increasing complexity of virtual graphic worlds. Huge models are produced in a number of applications, e.g. terrain modeling, volume visualization, virtual reality, automatic modeling based on range scanners, free form surface modeling. Models are usually specified with [triangle-based] surface meshes, and nowadays a key issue is how to store, access and visualize in real time hundreds of thousands of faces. Many methods for the simplification of geometrical information have appeared in the last few years. A direct consequence was the proposal of the Level of Detail (LoD) paradigm - a conceptual model where an object is stored through a constant number $k$ of different representations, each of them at a different level of detail or approximation [5]. LoD are usually adopted to speedup visualization, because simplified representations are sufficient for a large percentage of possible views [14, 18].

But LoD is neither the only nor the most sophisticated way to manage multiresolution. At this point we would like to use the term multiresolution (or continuous $L O D$ ) for only those data structures which allow the compact representation of a number $m$ of representations, at different levels of detail, where $m$ is not constant but is a monotone function of the data size (e.g., of the number of faces in the represented surface mesh). In other words, a multiresolution representation is not the simple collection of a [small] number of predefined models, but a compact representation of shape details plus the algorithms which allow an on-the-fly reconstruction of each possible level of detail representation [19, 2, 1, 31].

Multiresolution has been adopted at first to increase graphic throughput [14, 3], but different applications can be devised. Terrain visualization is one of the early fields of application of multiresolution modeling [7, 23, 4, 20, 10], e.g. to build dynamically variable resolution representations, which link resolution to the importance in viewing space of each projected parcel (e.g. a single terrain patch).

In this paper we try to extend the domain of possible applications. Rather than simply taking into account the viewing space "impact" of the represented data, a variable resolution representation of an object can be conceived as a user-driven interpretation on the object itself, optimized to convey a given amount of knowledge. For this reason we believe that resolution modeling has a strong similarity with shape modeling, in the sense that it has to be fulfilled through a tight interaction with the user. While the construction of the multiresolution representation is a process which can be simply made in an automatic and unattended way, the resolution modeling phase generally involves an interpretation of the data which cannot be fulfilled without human intervention.

Given this framework, the rationale of this paper is to propose a new data structure and algorithms which allow the user to add/remove resolution to localised parts of a model. Standard CAD systems provide tools to assist users in the design of "shapes", but none of them actually provide the tools needed to manage what we would conceive as a second-stage modeling session: given a first stage in which the "shape" is designed/scanned in full detail, then we want to allow the user to play with resolution to build different instances of the the input shape which are characterised by variable resolutions/details. Our global modeling conceptual model is therefore characterised by three phases as follows:

1. shape modeling, the canonical three-dimensional shape modeling (via CAD design, range scan acquisition or isosurface fitting);

2. multiresolution model construction, recent surface simplification methods $[2,19]$ easily support this phase; 
3. resolution modeling, user-driven modeling of variable resolution representations of the given shape.

Our goal is therefore to design an interactive tool which provides the user with selective and incremental resolution modeling features. This tool, called Zeta, adopts a multiresolution representation for triangulated, orientable, 2-manifold surfaces in 3D space, which allows compact storing and efficient navigation over a multiresolution mesh. We propose with Zeta a new methodology for the unified management of selective refinements and selective simplification (i.e., either increasing or reducing the mesh detail locally on mesh subareas chosen by the user). This selective refinement/simplification operator has been implemented via the efficient navigation over the multiresolution representation, which allows partial mesh updates and guarantees $\mathcal{C}_{0}$ continuity (i.e. no cracks) on each intermediate variable resolution mesh.

In the Zeta system framework, resolution modeling is achieved with the following steps:

LOAD a multiresolution representation of the mesh;

SELECT a representation at a fixed level of resolution;

LOOP

[ user: select the radius of the current region of interest ]

[ user: toggle between refinement/simplification; ]

[ user: modify the current Error propagation function; ]

user: pick the mesh to select the current action focus point;

Zeta: perform a selective refinement/simplification action on the

current variable resolution mesh;

UNTIL completion of variable resolution mesh modeling.

\section{Resolution Modeling Applications}

We believe that resolution modeling is a very general methodology. Consequently, the potential application domain is broad, and in the following we only glance to some possible uses. We subdivide resolution modeling applications into two broad classes: those where resolution modeling can be managed in an unattended, computer-driven manner, and those which have to be operated under strict user control.

Computer-driven applications have been recently proposed, to construct variable resolution terrain models for flight simulators [7, 23, 20, 4], or to build dynamic LoD [31, 20]. Variable resolution meshes are produced in this applications by taking into account the current view specifications.

Much less studied are the possible user-driven applications, such as:

- the editing of shapes acquired via range scanners or other automatic acquisition devices/algorithms. Direct acquisition produces highly detailed shapes and the standard simplification codes operate on these meshes using the same criterion on the whole mesh, generally a user-defined approximation threshold. But real applications may need to apply a variable threshold on the mesh. Let us consider human body acquisition: high precision is generally required for the face or the hands of the subject (perhaps in the range of one millimetre or less), while a much larger threshold may be imposed on less important areas such as the legs or the torso;

- the design of characters in computer-based animation. Each single character may be specified in terms of $n$ different variable resolution representations, which are built to take care of different presentation contexts (e.g. a foreground character which is either half length or full length); 
- the production of assembly instructions for 3D compound objects or systems. To produce illustrations or animated sequences of an assembly, often the entire and highly complex description of each subcomponent is not needed or even counterproductive. A variable resolution representation may be adopted to convey a complete description of only the surface section that play a major role in the current assembly action, therefore enhancing the cognitive importance of the most detailed part and reducing both image cluttering and rendering times. An example of the use of two different rendering modes, to enhance the different information given to the user, is presented in Figure 12; the choice among the two rendering modes (shaded or edge only) is driven by surface resolution.

Paper organization. Section 2 briefly reviews the state of the art in surface simplification and multiresolution representation. Section 3 describes how a multiresolution model, i.e. the Zeta input data, can be constructed, starting from classical incremental simplification or refinement approaches. The innovative contributions of the paper are presented in Section 4: we introduce the compact and efficient multiresolution data representation scheme (Subsection 4.1), its initialization for multiresolution data loading (Subsection 4.2), and the selective refinement/simplification algorithm (Subsection 4.3). Simple features for editing the multiresolution mesh are sketched in Section 5. An evaluation of the proposed methodology is presented in Section 6. Finally, conclusions are drawn in Section 7.

\section{Previous work}

Many approaches have been proposed to reduce the complexity of surface meshes [24]. They may be classified, at a high level of abstraction, into two main classes. Refinement heuristics start from a model (usually based on a simplicial complex) whose vertices are a very small subset of the input dataset $S$ and iteratively insert vertices in the mesh, until the model satisfies the required precision [13]. Simplification heuristics start from the input model and iteratively reduce complexity, by discarding as many elements as possible, while maintaining the required precision. Many different simplification approaches have been proposed, based on mesh decimation [28, 6, 25, 2], mesh optimization [21, 19], re-tiling [29], multiresolution analysis [11], and vertex clustering $[26,16]$.

An LoD representation may be simply built by the iterative application of any of the simplification/refinement approaches above, while only few of them propose the construction of multiresolution mesh representations [19, 2, 11]. The LoD paradigm has been incorporated in recent libraries or toolkits for 3D graphics, e.g. OpenInventor and VRML, and in some solid modellers or scene editors. The inherent limitations with the current implementations of the LoD paradigm are the limited number (generally very few) of different approximations which are stored, to reduce redundancy and space occupancy, and the non dynamic nature of the representation itself. The represented levels are generally built in a preprocessing step, to allow the fastest access to data in rendering. But the selection of the resolution would ideally depend on dynamic parameters, e.g. to ensure data-independent constant frame rates. This is obviously the case of digital terrains visualization; multiresolution digital terrain models have been reviewed in a recent paper by De Floriani and Puppo [8]. Methods for the construction of multiresolution representations of generic surfaces in $\mathbb{R}^{3}$ have been proposed by adopting classical face-based approaches $[31,20,2,32]$ or wavelet-based approaches $[11,17,1]$.

A seminal work on local refinement and multiresolution modeling, which somehow anticipated what we propose in this paper, was proposed by Forsey and Bartels [12]. They designed a system for locally refining surfaces represented with hierarchical B-splines. 
LoD or multiresolution representations have been adopted in many applications: to reduce rendering time in visualization $[14,3,30,27,7,31,4,8,23,10]$; to apply progressive transmission of 3D meshes on low bandwidth lines [19, 1]; to implement selective refinements or multiresolution editing on surfaces $[7,19,20,32]$.

In particular, multiresolution data structure have been proposed to allow the dynamic extraction of view-dependent variable resolution meshes. Xia and Varshney [31] proposed the merge tree structure which encodes in a binary tree all of the allowed vertex collapse actions and the dependencies between these actions. To extract a variable resolution mesh, the merge tree is visited recursively bottom-up to find all of the vertices that have to be included in the output mesh (e.g. by evaluating a criterion based on the size in view coordinates of the split edge). The set of triangles corresponding to these vertices may be computed from scratch by starting from the high resolution mesh and progressively collapsing edges, or by computing the current mesh $M_{i}$ from the mesh $M_{i-1}$ taking into account frame-to-frame coherence.

Whereas the merge tree representation is based on edge lengths and constrain the hierarchy to a set of levels with non overlapping collapsing fragments, the Hoppe's progressive meshes approach [19, 20] lets the hierarchy be formed by an unconstrained, geometrically optimized sequence of vertex splitting transformations, and introduces as few dependencies as possible between these transformations, in order to minimize the complexity of the extracted meshes. On this data structure, Hoppe defines a dynamic refinement function which determines whether each single vertex has to be refined, based on current view parameters (a refinement is applied only if the vertex to be split is in the view frustum, if adjacent faces are not back-oriented, and if the associated screen-space geometric error is higher than a predefined tolerance).

An interactive multiresolution mesh editing approach [32] was proposed with an objective somehow similar to our: to increase usability and efficiency of a modeling system by adopting a multiresolution representation of the meshes. The user manipulates high resolution geometry as if working in a patchbased system, with the additional benefit of hierarchical editing semantics. Using sophisticated adaptive subdivision techniques coupled with lazy evaluation, a scalable editing system has been defined. Through the use of subdivision and smoothing techniques the system supports large scale smooth edits as well as tweaking detail at the individual vertex level, while maintaining a concise internal representation (based on a forest of triangle quadtrees). But because the representation is based on quartic triangle refinements, an inherent limitation of this approach is that the input mesh must possess subdivision connectivity; otherwise it has to be re-meshed.

Unlikely previous multiresolution data structures [31, 20,32], our multiresolution scheme is the only one which explicitly stores topological information between the fragments that compose the multiresolution mesh, and which allows "walking" on the multiresolution surface, moving efficiently between adjacent faces (which may have the same or a "compatible" approximation error).

\section{Construction of a multiresolution model}

A surface mesh $S$ may be simplified by following either an incremental refinement or simplification strategy. In both cases, a multiresolution output may be simply built if a global error measure is evaluated after each local modification action. In the following we take into account a simplification heuristic based on vertex decimation, but the same holds for other incremental simplification or refinement heuristic as 
well.

Given a simplification approach based on vertex removal, we call: $S$, the input mesh; $S_{i}$, an intermediate mesh obtained after $i$ steps of the simplification process; $v$ the vertex candidate for removal on mesh $S_{i}$; $T_{v}$ the patch of triangles in $S_{i}$ incident with $v$; and, finally, $T_{v}^{\prime}$ the new triangulation which will replace $T_{v}$ in $S_{i+1}$ after the elimination of $v$.

At each step, we evaluate the global error of the current mesh $S_{i}$, with respect to the original input mesh $S$. Incremental simplification approaches which adopt a global estimate of the error are available $[2,22,25,16]$.

If we consider all surfaces built at intermediate simplification steps, we have a whole sequence of triangulations $\left\{S_{0}, \ldots, S_{n}\right\}$, where $S_{0}$ is the input triangulation $S$, and $\forall i=0, \ldots, n$, the surface $S_{i}$ approximates the full resolution mesh with an error $\varepsilon_{i}$. The only constrain imposed on the simplification code is that the sequence of error tolerances $\varepsilon_{i}$ should increase monotonically: $\varepsilon_{0}=0<\varepsilon_{1}<\ldots<\varepsilon_{n}$. Moreover, the slower and smoother the growth of the approximation error is, the larger are the number and the quality of the different resolution meshes stored in the multiresolution model [2].

Let us consider the set $T^{*}$ of all the triangles that were generated during the entire decimation process, including the triangles of the original mesh. Each facet $t \in T^{*}$ is characterised by two errors: error at creation time (or birth error, the error of the current mesh $S_{i}$ when $t$ was generated as part of a new patching sub-mesh) and error at elimination time (or death error, the error of the current mesh $S_{i}$ when $t$ was found as one of the triangles incident on a vertex being removed). Each facet $t \in T^{*}$ is therefore tagged by these two errors $\varepsilon_{b}$ and $\varepsilon_{d}$, with $\varepsilon_{b}<\varepsilon_{d}$. The interval bounded by these two errors is called life interval.

A straightforward multiresolution representation of the output produced by a simplification algorithm is therefore the list $T^{*}$, with the $\varepsilon_{b}$ and $\varepsilon_{d}$ errors associated to each facet $t \in T^{*}$. This historical representation, called history for short, consists of two lists:

- vertex list - for each vertex $v_{i}$, its $[\mathrm{x}, \mathrm{y}, \mathrm{z}]$ coordinates;

- face list - for each face $f_{i}$ : three indices to its vertices and its life interval $\left[\varepsilon_{b}, \varepsilon_{d}\right]$.

The history avoids the replication of all the triangles which belong to more than one fixed resolution mesh (or layer), and yelds an improvement in terms of memory space occupancy over the layered models (e.g. LoD or other hierarchical layer-based structures). A prototypal decimator [2] which returns in output a multiresolution mesh and uses the history data format is available on the web ${ }^{1}$.

The extraction from the history of a representation $S_{\varepsilon}$ at a given precision $\varepsilon$ is straightforward: $S_{\varepsilon}$ is composed of all of the faces in the history such that their life interval contains the error threshold searched for $\left(\varepsilon_{b} \leq \varepsilon<\varepsilon_{d}\right)$.

But the history representation is not sufficient when more sophisticated accesses to the multiresolution data have to be managed. To support the efficient implementation of a selective refinement operator, a more sophisticated multiresolution mesh representation is proposed in the following section.

\section{Resolution Modeling}

This section describes the data structures and the kernel functionalities of Zeta, our prototypal resolution modeling system. The key functionality is to support selective refinement or simplification actions. Each

\footnotetext{
${ }^{1}$ See URL: http://miles.cnuce.cnr.it/cg/enhadecimation.html
} 

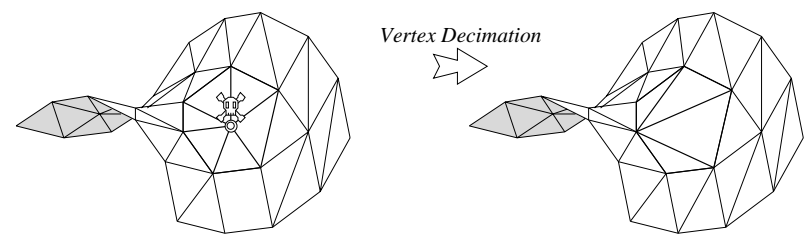

Patch Glueing
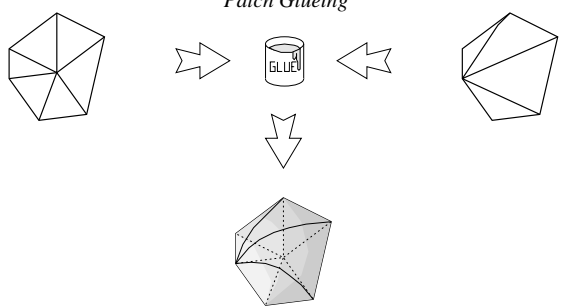

Figure 1: The Hypertriangulation (HyT) multiresolution scheme: the patches associated with a single simplification step (based on vertex decimation) are "glued" onto the common border.

action modifies incrementally the current mesh by increasing/decreasing the resolution on a sub-area of the mesh which is interactively selected by the user. It has to be performed in interactive time, and it must guarantee $\mathcal{C}_{0}$ continuity on each intermediate result (the current output mesh).

Being the process user-driven, a complex dialog session has to be managed. The user has control over:

- the focus point $p_{f}$ on the current mesh;

- the current radius $r$, which identifies the area size (to be refined/simplified) surrounding $p_{f}$ on the current mesh.

- the action (refinement or simplification) that has to be operated and the error function $E$ which determines, for each element of the mesh, the required increase/decrease of precision by taking into account the distance of the element from $p_{f}$.

According to user inputs, the system modifies locally the current mesh, by decreasing or increasing the mesh precision in the mesh subsection of radius $r$ and centred in $p_{f}$.

The interface of Zeta ${ }^{2}$ is presented in Figure 8. Six different stages of a resolution modeling session are presented in Figure 9, to highlight some of Zeta's capabilities. The mesh colors in the second, third and fourth clips represent the error of each mesh face (using a color ramp from blue to red).

\subsection{The Hypertriangulation scheme}

This section introduces a multiresolution scheme for orientable, 2-manifold surfaces in $\mathbb{R}^{3}$, called $\boldsymbol{H} \boldsymbol{y}$ pertriangulation $(H y T)$ [4]. The scheme is general and can be adopted to store multiresolution output produced either by simplification or refinement algorithms. For the sake of simplicity, hereafter we will only consider simplification algorithms.

We start from the history (see Section 3), and build a new representation to encode explicitly the adjacencies between facets which share an edge, either in the same triangulation $S_{i}$ or in different triangu-

\footnotetext{
${ }^{2}$ The first release of Zeta is available on the World Wide Web at address http://miles.cnuce.cnr.it/cg/zeta.html
} 

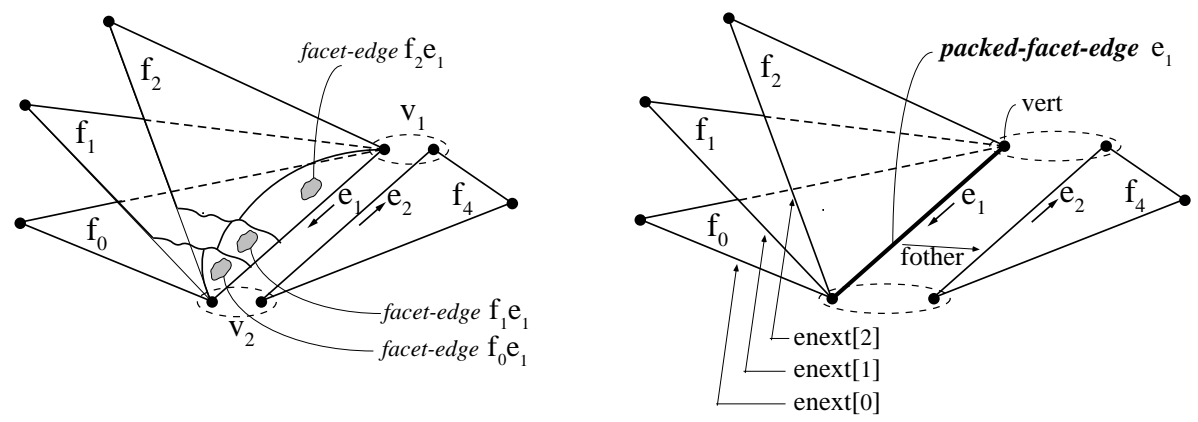

Figure 2: On the left, the original implementation of the HyT based on facet-edges, (one facet-edge record for each face-edge pair); on the right, the new packed facet edge representation (a single record for all of the facets on the same side of the oriented edge).

lations. Single triangulations (i.e. meshes at fixed approximation $\varepsilon_{i}$ ) are not explicitly and independently stored in our structure, but we provide tools to reconstruct them efficiently at run time.

Let us consider the refinement region that is simplified in passing from $S_{i-1}$ to $S_{i}$. The simplification action operated onto $S_{i-1}$ defines two patches: the removed patch, i.e. the triangles in $S_{i-1}$ incident on the vertex to be removed; and the new patch, i.e. the triangles which re-triangulate the resulting hole. By definition, the two sets of triangles share the edges that bound the current simplification region. Hence, instead of simply replacing the removed patch, we "glue" along such boundary edges the new patch over the old one. In order to clarify the organization of the data, let us use a metaphor: we represent "visually" the adjacency between patches by representing a new patch as a curved bubble which shares with the removed one the chain of border edges (Figure 1). We can imagine that the resulting multiresolution data structure is built by warping each new patch of a delta value sufficient to contain the removed patch, and by welding it onto the old triangulation at the boundary of the old patch. The single simplification step is therefore visually represented as a new "bubble" glued onto the old patch.

The resulting structure can be topologically (but not geometrically) interpreted as a 3D subdivision of the space (where a 3D cell corresponds to each bubble). This because geometry (i.e. vertices coordinates) is not modified at all.

The HyT multiresolution scheme follows the metaphors above. It maintains in a compact format both the topological information, collected during the refinement process, and the information on the error of each triangle. The HyT scheme is encoded by adopting a packed facet-edge (PFE) representation. With respect to its original design [4] (based on the facet-edge representation), the current HyT implementation is more compact and more efficient, because the lower redundancy allows faster implementation of critical traversal functions.

The PFE has been designed as a modification of the facet-edge, a data structure originally introduced for the representation of 3D space subdivisions [9]. In the facet-edge scheme (see Figure 2.left), an atomic entity is associated with each pair that is identified by a face $f$ and one of its edges $e$ : the so-called facet-edge. Each facet-edge denotes two rings: the edge-ring, composed by all the edges of the boundary of $f$; and the facet-ring, composed by all the faces incident at $e$. This structure is equipped with traversal functions that enable the complex to be visited. These functions are used to move from a facet-edge to an adjacent one, either by changing edge or by changing face (note that since our multiresolution model 
is topologically equivalent to a 3D subdivision, more than two faces may be incident at each edge).

Instead of having a single facet-edge for each edge-face pair [4], the PFE representation encodes into a single record all of the facet-edges incident on a given oriented edge from one of its sides (see Figure 2.right). Each PFE stores both geometrical and topological information related to the oriented edge and to the faces sharing it. The data structures that implement the HyT scheme are therefore composed of three entities: the Vertex, the Face and the PackedFacetEdge record.

The Vertex record holds the vertex coordinates plus two more fields (the distance of the vertex from the current focus point and a mark field), whose usage will be clarified in the following.

The Face record holds the life interval of the associated face ( $\varepsilon_{b}$ and $\varepsilon_{d}$ values) and a mark field, which is used as an incremental counter by the Selective Refinement algorithm.

The PackedFacetEdge is the main entity. It stores: the index of the starting vertex of the oriented facet-edge (vert field) and to the adjacent oriented facet-edge (fother field); and, for each of the faces piled up on the oriented edge, a pointer to the next PFE in the edge ring (enext field) and a pointer to the associated Face record (face field). PFE are not replicated: if $k$ faces share the same oriented edge, then we have only one PFE which represents all of these edge-face pairs. The PFE is a variable length record, due to the non constant number of faces which may be incident on the same edge, and it is defined in pseudo-code as follows:

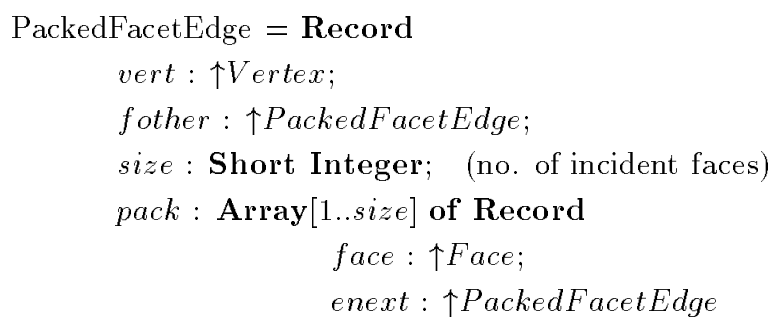

The actual size of each oriented edge $e$ depends on the number of simplification steps which have $e$ on the border of the simplification region. The incident faces are ordered in the variable length vector upon increasing error (as in the metaphor of the overlay of bubbles). Following the definition of the approximation error, the life intervals of two successive faces in a PFE have to be adjoining, and therefore the total life interval of a $\mathrm{PFE} e$ is delimited by the birth error of its first incident face and the death error of its last face. Moreover, by construction the life interval of a PFE is identical to the life interval of the adjacent one (linked by the fother field).

Basic access functions are provided in our implementation of the HyT scheme to compute properties or adjacency relations which are not explicitly stored. In particular, we define: Life_Interval(e), which returns the total life interval of a PFE; Star $(e)$ which, given a PFE $e$ and its vertex $v$, returns the set of vertices which are adjacent to $v$ on the maximal resolution mesh; FindValidFace $(e, \varepsilon)$ which returns the face incident on a given PFE which satisfies approximation precision $\varepsilon$.

The traversal of the HyT data structure is implemented by alternating two different moves: traversing in the topology domain and traversing in the error domain. In the first case, given an oriented PFE we want to move to the adjacent one on the opposite side of its oriented edge (through the fother link). In the latter, given an error, we want to adjust the precision by moving in the pack list of faces stored in the current PFE record. The structure proposed for the HyT scheme has been designed to allow an efficient implementation of these two main traversal modes. 


\subsection{HyT model construction}

The HyT representation may be built either during simplification, or as a post processing phase by converting the simplification results (coded using the history representation) into a $H y T$ model. We will briefly describe here the second approach, because it allows us to convert the output of different simplification approaches into the $H y T$ representation.

The history_to_HyT conversion algorithm consists of the following steps.

Vertex and Face record lists initialization: storing of vertices coordinates and facets life intervals into the Vertex and Face records; each face is oriented (counterclockwise).

Edges identification: a temporary list of edges is built, with pointers (indices) to the incident faces associated with each edge.

Ordered edges detection and PackedFacetEdge construction: for each edge, the list of incident faces is processed: we split the list into two sets (each containing the faces on the same side of the edge); each set is sorted taking into account face life intervals and then allocated into a PackedFacetEdge record; all the topological links are then suitably initialized.

Optimization of the representation to reduce access time: it is very likely that facets with similar life intervals will be accessed in close times. To reduce page/cache faults and swapping, we sort the Vertex, Face and PackedFacetEdge vectors taking into account the lower bound of their life interval. All records with similar life intervals will thus be allocated, with a sufficiently high probability, into neighboring memory chunks. We proved empirically the validity of this simple criterion, and measured significant improvements in times.

\subsection{Selective Refinement/Simplification on the $H y T$ scheme}

In this section we present the selective refinement/simplification algorithm. As outlined at the beginning of Section 4, the user-machine interaction starts with the selection of a constant resolution representation of the mesh (see Figure 9). Therefore, we introduce first the algorithm to extract a constant resolution mesh out of the HyT scheme. Then, we describe the approach chosen to compute distances on the mesh, which are needed to select the area onto which each selective refinement action has to be operated. The selective refinement algorithm is specified in the last subsection.

\subsubsection{Extraction at constant approximation}

The constant approximation extraction is similar to the one proposed for terrain multiresolution representations [4]. Each intermediate triangulation $S_{i}$ can be retrieved by visiting the $H y T$, starting from a $\mathrm{PFE} e \in S_{i}$ ( $e$ is by selection a PFE which contains the precision $\varepsilon$ in its life interval) and propagating from $e$ by means of the PFE adjacencies. To implement efficiently the search of this initial PFE we build off-line a small, not optimal subset Seeds of PFE's such that the union of their life intervals should be equal to the global error range of the $H y T$ model. By construction, for each error $\varepsilon$ we will find, simply by scanning the Seeds set, an initial PFE which satisfies the required precision. The Seeds set results in a few tens of elements, as we evaluated empirically onto multiresolution meshes composed by $200 \mathrm{~K}$ faces and $10 \mathrm{~K}$ different error values.

Then, this first PFE is inserted into a stack together with the adjoining PFE (the one pointed by the fother field), and the mainloop is started: we extract a PFE from the stack, choose from the incident faces the 

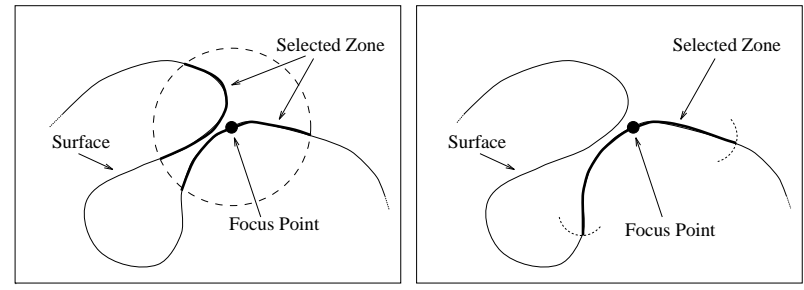

Figure 3: Euclidean distance on the left, and geodetic distance on a surface on the right.

one $\left(f_{j}\right)$ that satisfies error $\varepsilon$, insert this face in the output mesh $T$, and finally insert in the stack the two PFE's adjacent to the two other edges of $f_{j}$. The loop terminates when the stack is empty.

\subsubsection{Distance on a surface: definition and evaluation}

The evaluation of distances on the multiresolution mesh is required, because we want to limit the action of each selective refinement/simplification to a mesh portion surrounding the user-defined focus point $p_{f}$. Therefore, we must determine which faces are at distances less than $r$ (the radius selected by the user) from $p_{f}$. Distances on a surface in $\mathbb{R}^{3}$ cannot be computed in terms of Euclidean distances (see Figure 3 ), but geodetic distances are needed; to simplify computations, we used approximated geodetic distances:

Definition 4.1 The geodetic distance of two points on a surface $S$ is the length of the minimal curved arc which lies on $S$ and connects the two points. The approximated geodetic distance of two vertices on a triangulated surface $T$ is the length of the minimal path, composed by the edges of $T$, which connects the two points.

To define the distance between two vertices $v_{1}$ and $v_{2}$ in a multiresolution model, we take into account the mesh at maximal resolution $S_{0}$, which gives the best approximation of the geodetic distance. The precision of the approximated geodetic distance depends on the regularity of the tessellated representation. If the mesh is composed of equilateral triangles, then the approximation is at most $2 / \sqrt{3} \cong 1.154$ times the precise geodetic distance.

To compute approximated geodetic distances we consider a triangulated mesh as a weighted graph, whose arcs are the mesh edges and whose arc weights are the length of the associated edges. We need to compute the minimal distances from the focus point, i.e. to solve a Shortest Path Tree (SPT) problem with a single source. A number of standard solutions exist; we adopted a modified version of the SPT_S_Heap algorithm $[15]$.

All of the vertex distances computed are stored in the HyT model (in the field dist of the Vertex records).

During the design of the Zeta GUI, we discussed for some time on how to show to the user the current extension of the focus area, during the selection of the radius length. The effective extension of the focus area depends on both the current radius and the current focus point, and therefore it can't be determined before these to parameters have been set. But we wanted to separate the selection of the radius (which is generally selected once and then used for multiple refinement/simplification actions) from the selection of the current focus point. For this reason, we chose to provide the Zeta GUI with a visual feedback during the radius length selection, which shows to the user an approximated (and focus-point independent) focus area. We decided to display interactively a transparent sphere (centered on the last focus point), which 
only shows the magnitude of the current radius and is not related to the actual geodetic distance.

\subsubsection{The Selective Refinement/Simplification algorithm}

The selective refinement/simplification algorithm locally modifies the current mesh $T$, which can be either a surface extracted at constant error from the $H y T$ model, or the composition of $n$ subsequent selective refinements.

Selective refinement/simplification parameters (set by the user) are:

- the current point of focus $p_{f}$;

- the radius $r$ of the current selective update;

- the error function $E: \mathbb{R}^{+} \rightarrow\left[\varepsilon_{1}, \varepsilon_{2}\right]$ which sets the error expected for each element $f_{i}$ of the mesh in the focus region as a function of the distance between $f_{i}$ and the focus point $p_{f}$; it is defined in the interval $\left[\varepsilon_{1}, \varepsilon_{2}\right]$, with $\varepsilon_{1}$ the approximation error that must be verified by faces at distance zero from $p_{f}$ and $\varepsilon_{2}$ the one for faces at distance $r$.

A refinement or a simplification action is performed depending on the current selection for the error function $E$ domain: if $\varepsilon_{1}<\varepsilon_{2}$ than the user requests a local refinement (approximation must be higher in the point of focus than in the focus region boundary), otherwise a local simplification has to be executed. Obviously, if the focus region is entirely at approximation $\varepsilon<\varepsilon_{1}$, then no selective refinement is applied. In the current implementation, the user may interactively define the current function $E$ by editing its graph in the Error Function section (bottom-right of the Zeta main window, see Figure 8).

Given a the current mesh $T$, the Selective Refinement algorithm builds progressively a new mesh subsection and updates $T$. Starting from the facet edge nearest to the focus point, it starts a topologic expansion by following the adjacency links and choosing the next faces by taking into account the $E$ function and the distance from the focus point. The topological expansion is implemented by using a priority queue, which encodes the facet-edges which have to be expanded, and it is limited by both the radius set by the user and the need to maintain connectivity between the refined region and the mesh $T$ to be updated. At the end, the current variable resolution mesh is updated by removing the mesh section which has been replaced by the refined one.

Specifically, the main steps of the Selective Refinement algorithm are:

- select the PFE $f e$ which is nearest to $p_{f}$ and satisfies the precision $\varepsilon_{1}$ required for the focus point (FindFirstEdge function);

- initialize the priority queue by inserting in it both fe and its adjacent fe.fother;

- compute geodetic distances from $p_{f}$ (SPT_S_Heap function);

- initialize to null the overlapStack, i.e. the data structure that will hold all the facet-edges on the border of the refined mesh section;

\section{- main loop:}

- extract the next PFE fe from the priority queue; 


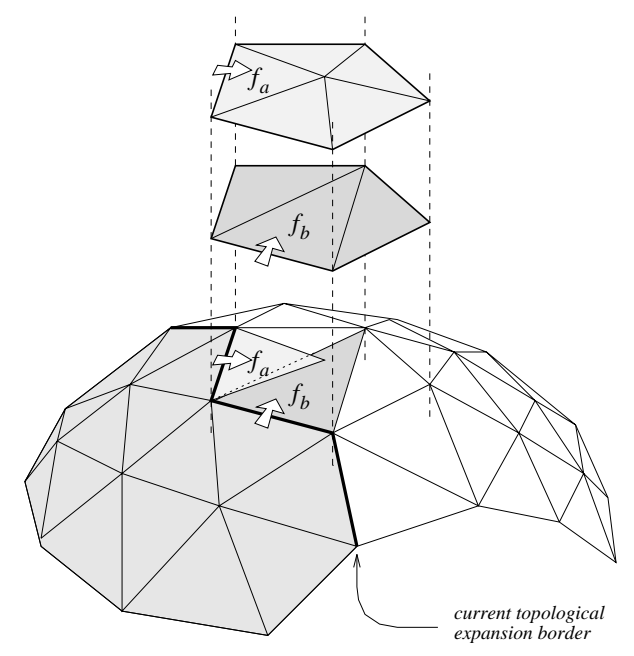

Figure 4: An inconsistency which has to be prevented in variable approximation extraction: given two different expansion paths $\left(f_{a}\right.$ and $\left.f_{b}\right)$, the associated sub-meshes do not match.

- if $f e$ is further than $r$ from $p_{f}$ and $f e$ is already contained in $T$, then we have found an edge which reconnects the new refined region with the previous variable resolution mesh; fe is therefore not expanded further and is inserted into the overlapStack data structure, which will be used later on to update $T$;

- otherwise, expand $f e$ and detect the face $f$ which satisfies the current error; then insert $f$ it into the current output mesh $T$; for each of the other two edges of $f$, insert them into the priority queue without replication (i.e., if the edge $f e$ ' is already contained in the priority queue then remove it; otherwise, insert fe' fother in the priority queue);

- update the output mesh $T$ by removing all of the facets that have been replaced with the new refined section (the overlapStack contains the border of the section which has to be removed).

FindFirstEdge function. The PFE nearest to the focus point which satisfies the error $\varepsilon$ is returned by the FindFirstEdge function. To guarantee efficiency, it has been implemented by adopting a spatial index on the PFE's (organised by partitioning the space into rectilinear sub-volumes). For each point in the space, FindFirstEdge retrieves efficiently a subset of PFE which are in the proximity of $p_{f}$, by visiting only a subset of the cells of the spatial indexing data structure. Then, starting from this set, it computes the vertex nearest to $p_{f}$. Finally, it chooses from the PFE's incident in the nearest vertex the one which is also nearest to $p_{f}$.

Priority queue management. The order of extraction of the PFE's from the priority queue is critical for correctness and result quality of the Selective Refinement algorithm. If we extract PFE's using a random strategy, then inconsistent expansions might be produced. See an example in Figure 4, where the independent expansion of two PFE originates a not joining mesh section. In this case, the same surface sub-area is represented by two different faces $\left(f_{a}, f_{b}\right)$, which [partially] overlap once projected to the original high-resolution surface. But, by construction of the multiresolution model [2], if two faces 
represent (at least partially) the same original surface area, then their life intervals must be disjoint. The case in Figure 4 can be prevented if we expand facet-edges by taking into account the global current content of the priority queue. In particular, we impose the constraint that for all the facet-edges on the priority queue the intersection of their life intervals has to be not empty. The inconsistency above is therefore prevented, because as stated previously if two faces in the $H y T$ model cover the same portion of the original surface, then, by construction, their life intervals have to be disjoint. In other words, facet-edge expansion and insertion on the priority queue have been designed so that in each instant an $\varepsilon$ value exists such that $\varepsilon \in \operatorname{LifeInterval}\left(f e_{i}\right)$ for all facet-edges $f e_{i}$ in the priority queue. This implies that the expansion of each facet-edge is driven by the user-selected error function, but takes also into account the status of the priority queue: a too much rapid decrease/increase of the error, such that the selected error $\varepsilon_{i}$ will be not contained in the current priority queue's life interval, is denied and clipped to the nearest extreme of the current priority queue's life interval.

This condition imposes a limitation on the ability to reduce the error following the error function (let us again assume we are executing a refinement action). In the case of a facet-edge with several possible adjacent faces, we may be limited in the choice of the next face by the current status of the priority queue: the best fit face, according to the distance-based error function, may result in the choice of an error value which is not contained in the current intersection of all life intervals currently stored in the priority queue. Let us introduce some more terminology to clarify the approach: given a priority queue $Q$ and the set of life intervals of all the facet-edges $e_{i}$ stored in $Q$, the intersection of all the life intervals is delimited by:

$$
Q . \varepsilon_{b}^{\max }=\max _{e \in Q} e . \varepsilon_{b}, Q . \varepsilon_{d}^{\min }=\min _{e \in Q} e . \varepsilon_{d} .
$$

Therefore, for each new expansion we need to test if the proper distance-based error is contained in the priority queue current interval $\left[Q \cdot \varepsilon_{b}^{\max }, Q \cdot \varepsilon_{d}^{\min }\right)$. The priority queue has to be implemented at least as a double heap, to hold efficiently the ordering of both the extreme values of the life interval.

A crucial point of the algorithm is now how to select the next PFE to be popped from the priority queue, and this choice has a strong impact on the quality of the variable resolution mesh extracted. As stated above, to ensure the validity of the mesh extracted it is mandatory to search for a face at an error included in the interval $\left[Q . \varepsilon_{b}^{\max }, Q \cdot \varepsilon_{d}^{\min }\right)$. If we extract from $Q$ adopting a naive selection criterion (e.g. FIFO), then the $\left[Q \cdot \varepsilon_{b}^{\max }, Q \cdot \varepsilon_{d}^{\min }\right.$ ) interval may become very narrow (in many cases it is reduced to a single value), thus preventing us from following the required error decrease/increase encoded by the $E$ function. To maintain this interval as wide as possible, we evaluated different priority queue management strategies:

- distance-based popping: we extract in order of distance (nearest first) from the focus point $p_{f}$; to assure efficiency, $Q$ has to be implemented as a triple heap, to provide a further sorting based on facet-edge distances;

- error-based popping: we extract the PFE whose error interval is origin of the bounds of the current priority queue interval; a side-effect of extracting that PFE is to widen the current priority queue life interval. If we are refining the mesh, i.e. extracting a sub-mesh with an error which increases as we get far from $p_{f}$, then we extract the facet-edge with $\varepsilon_{d}=Q \cdot \varepsilon_{d}^{\min }$; otherwise, if we are reducing resolution we select and extract the facet-edge with $\varepsilon_{b}=Q \cdot \varepsilon_{b}^{\max }$;

- composed distance-error based popping: we follow a distance-based approach until we can extract facet-edges at the proper error; then, we use the error-based criterion until the current priority 


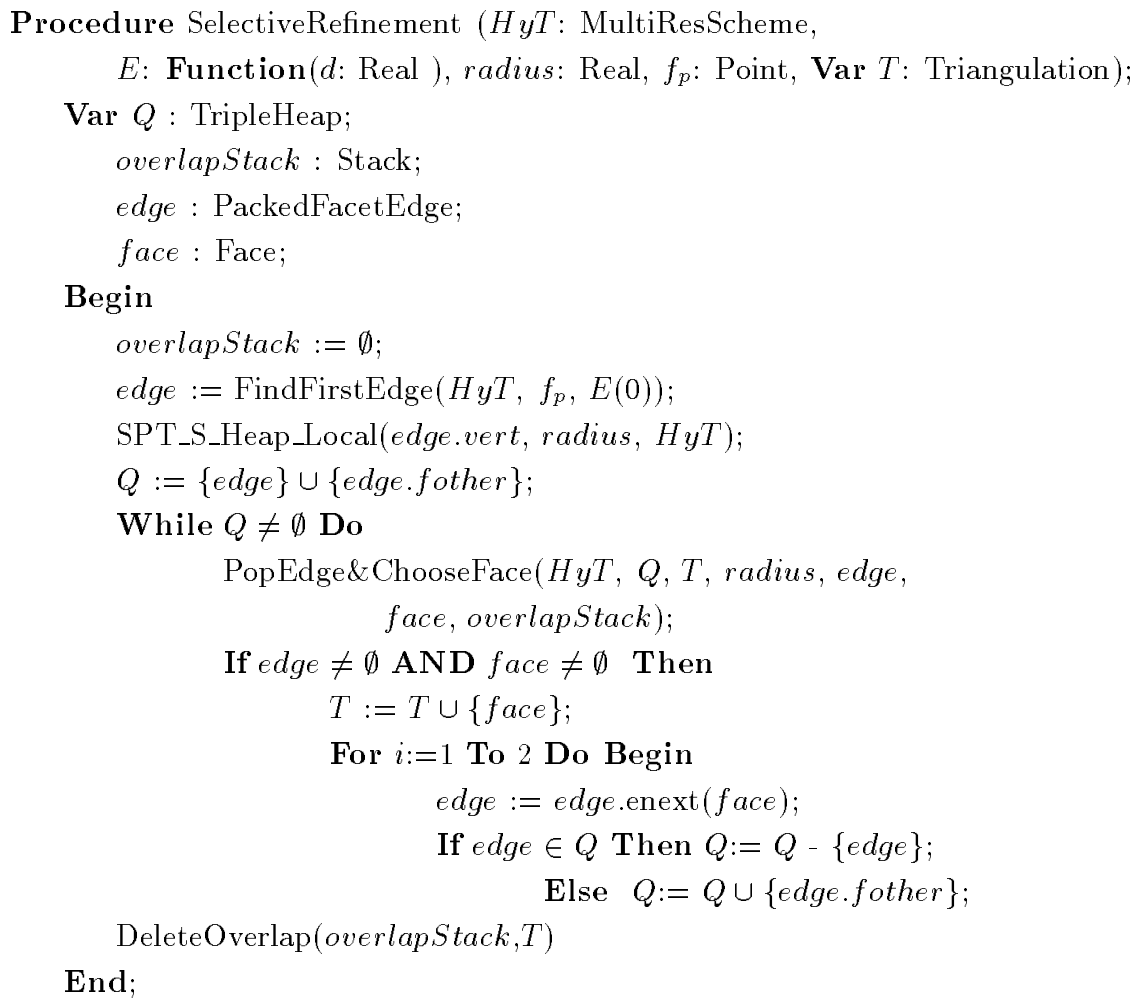

Figure 5: Selective Refinement pseudo code.

queue life interval has been sufficiently widened.

The previous strategies were evaluated on a number of datasets, and the composed distance-error strategy gave the best performances, in terms of quality of the extracted mesh. The composed strategy has been adopted in the Zeta prototype (see the pseudo code of Figure 6).

\section{Facet-edge expansion and mesh update}

For each facet-edge $f e$ popped from the priority queue $Q$, if the distance of $f e$ from $p_{f}$ is greater than the radius $r$ of the current selective update, and $f e$ is already contained in the current mesh $T$, then we do not proceed with the expansion of $f e$, and its adjacent facet-edge fe.fother is inserted into the overlapStack. If, conversely, $f e$ is farther than $r$ but is not contained in $T$, we cannot stop the expansion (to prevent cracks in the updated mesh $T$ ), and thus proceed with the expansion of $f e$.

In canonical conditions, at the end of the topological expansions the boundary of the section of the mesh $T$ which has been refined is stored in the overlapStack. The DeleteOverlap () function removes from $T$ the redundant faces: it starts from the facet-edges contained in the overlapStack and removes all of the faces which can be reached from these facet-edges and have a value of the field mark lower than the current refinement time (see Figure 7). The mark field of a Face record is a counter, whose value is initialized with the "time" of the selective refinement action that included such a face in mesh $T$. 


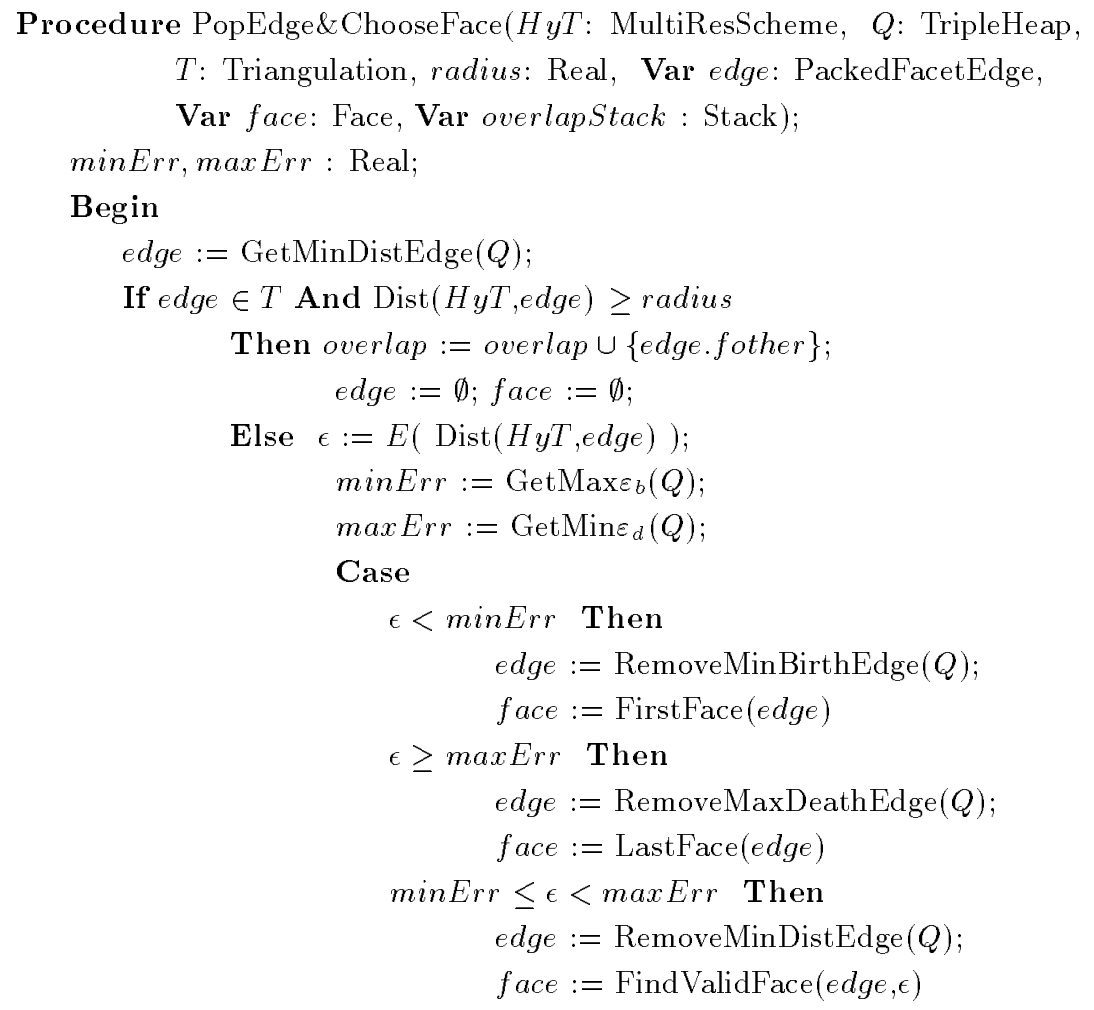

End;

Figure 6: Selective Refinement: selection and expansion of the next facet-edge from the priority queue.

Therefore, if a face $f$ can be reached from the oriented boundary stored in overlapStack and it has been inserted in $T$ as part of a previous refinement, then $f$ is part of the replaced patch and can be removed from $T$.

The difference between the focus area of the current selective refinement/simplification action and the actual size of the updated mesh section is shown in Figure 11. The colored mesh section represents the updated mesh region, and the color represents the geodetic distance from the current focus point. Faces rendered in white are those which were produced with distance higher than the current radius (i.e. out of the focus area), and which are needed to assure continuity of the variable resolution mesh.

\section{$5 \quad$ Multiresolution Shape Editing}

Simple shape editing features have been also included in the Zeta prototype, to show how mesh editing might be performed in a consistent manner on the multiresolution model. The goal is obviously not to support sophisticated functionalities (available in the shape editing framework), but to allow users to perform small changes to the shape of the model, without requiring to traverse again the entire pipeline (shape modeling, multiresolution construction, resolution modeling).

Shape editing actions are operated on the multiresolution scheme, and therefore shape changes are applied to every resolution. A shape editing session is started by selecting the Edit Shape option of the Edit menu. 

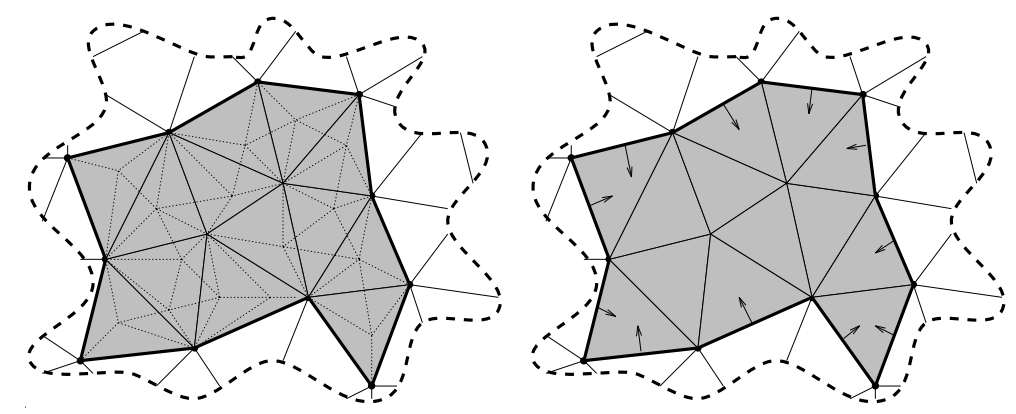

Figure 7: Updating the current $T$ mesh, after local refinement, from the oriented border of the refined region stored in the overlapStack.

User controls the mesh area where the current editing action will be applied with the standard Zeta approach (the focus zone depends on the current radius and on the current focus point). User starts with the selection of a proper (low resolution) representation of the mesh. Then, shape edit actions are performed in sequence and the results are automatically applied to every resolution and iteratively composed. Current radius and $E$ function determine the actual propagation over the mesh of each shape edit action.

Few snapshots out of a shape edit session are shown in Figure 10. The initial mesh is visualized in the top-left image. User can observe the result of the editing session at whichever level of resolution (full resolution is shown in Figure 10, bottom-right image).

\section{Results Evaluation}

\subsection{Space Complexity}

The mean value of the size field, which drives the variable length part of the Packed Facet Edge record, is needed to evaluate the space complexity of the HyT scheme. We call this value face adjacency factor $\eta$, defined as follows.

Definition 6.1 The face adjacency factor $\eta$ is the number of faces which are adjacent to a PFE; the mean face adjacency factor $\eta_{\text {mean }}$ is the mean of the $\eta$ factors for all the faces of a mesh.

The number of adjacent faces on each edge will depend on both the shape of the original surface and the simplification/refinement algorithm used to build the multiresolution mesh, and therefore it can only be determined empirically. Table 1 shows the number of faces with $\eta=1 . .8$ and their percentage on the total number of faces in the multiresolution mesh (obtained using the Jade code [2]).

Obviously, the larger $\eta_{\text {mean }}$ is, the better the results of the selective refinement algorithm will be, because if a larger number of different options on each edge is available then the correct value of the error given by the $E$ function will be available with higher probability.

The space complexity of the HyT scheme is the sum of the space required by the list of Vertex, Face and Packed Facet Edge records:

$$
\mathcal{C}_{\text {space }}(H y T)=5 * V+3 * F+\left(3+2 * \eta_{\text {mean }}\right) * E^{\prime}
$$




\begin{tabular}{|c|c|c|c|c|c|c|c|c|c|}
\hline & $\eta=1$ & $\eta=2$ & $\eta=3$ & $\eta=4$ & $\eta=5$ & $\eta=6$ & $\eta=7$ & $\eta=8$ & $\eta_{\text {mean }}$ \\
\hline \multicolumn{10}{|c|}{ Bunny mesh (vert.:34,834 faces:189,351 facet-edges:568,053) } \\
\hline number & 241,066 & 99,139 & 28,821 & 7,529 & 1,821 & 401 & 67 & 14 & 1.54 \\
\hline percentage & $63.6 \%$ & $26.1 \%$ & $7.6 \%$ & $1.9 \%$ & $0.4 \%$ & $0.1 \%$ & $0.01 \%$ & $0.003 \%$ & \\
\hline \multicolumn{10}{|c|}{ Sphere mesh (vert.:6,322 faces:33,801 facet-edges:101,403) } \\
\hline number & 43,788 & 18,427 & 4,744 & 1,114 & 322 & 63 & 11 & 1 & $\overline{1.48}$ \\
\hline percentage & $63.9 \%$ & $26.9 \%$ & $6.9 \%$ & $1.6 \%$ & $0.47 \%$ & $0.09 \%$ & $0.01 \%$ & $0.001 \%$ & \\
\hline
\end{tabular}

Table 1: Face adjacency factor evaluated on two different multiresolution meshes.

where $F$ is the number of non replicated faces in the multiresolution mesh (estimated empirically to be about 2.5 times the faces of the mesh at maximal resolution [2]) and $E^{\prime}$ is the number of non replicated oriented edges in the multiresolution mesh.

The number of oriented edges $E^{\prime}$ may be determined as a function of the number of faces $F$ and the mean face adjacency factor $\eta_{\text {mean }}$ :

$$
\eta_{\text {mean }}=\frac{3 * F}{E^{\prime}} \rightarrow E^{\prime}=\frac{3 * F}{\eta_{\text {mean }}}
$$

The overall HyT model size is therefore:

$$
\mathcal{C}_{\text {space }}(H y T)=5 * V+9 * F+\frac{9 * F}{\eta_{\text {mean }}}
$$

and according to a number of experiments the $\eta_{\text {mean }}$ on real meshes is in the range [1 .. 2].

In order to give an idea of the memory overhead which is due to the explicit representation of the topology in the HyT scheme, we report in Table 2 the size of two meshes, the Bunny ${ }^{3}$ and the Fandisk meshes (shown in Figures 9 and 12), considering both the representation of only the faces and the associated error intervals (i.e., the history format) and a complete geometry plus topology representation (the $H y T$ format).

The space complexity of other multiresolution data format is as follows.

The run-time size of the (non optimized) hierarchical selective refinement mesh representation [20] is $224 *|\hat{V}|$, with $|\hat{V}|$ the total number of vertices stored in the data structure (and $\hat{V}>V$, because for each edge collapse a new vertex is produced). Hoppe reported that the space occupancy of the bunny mesh is $7.8 \mathrm{MB}$ (and thus it is $72 \%$ of our $H y T$ representation).

Data on the space complexity have not been reported neither for the merge tree data structure [31], nor for the forest of triangular quadtrees [32].

\subsection{Time Complexity}

The history_to_HyT scheme conversion requires times in the order of few tens of seconds on complex meshes (i.e. to convert history files with $\cong 100 \mathrm{~K}$ faces), but it might be considered as a pre-processing phase. The Zeta system may also directly save onto disk a multiresolution mesh encoded in $H y T$ format.

The asymptotical time complexity of the Selective Refinement algorithm depends directly on the number of faces returned by each refinement action: the main loop is executed once for each face produced, with a constant number of insertions/extractions from the priority queue (performed in logarithmic time).

\footnotetext{
${ }^{3}$ The Bunny model was acquired automatically with a range scanner and distributed by M. Levoy at Stanford Univ.
} 


\begin{tabular}{|c|c|c|}
\hline & Bunny mesh & Fandisk mesh \\
\hline vert. & 34,834 & 6,475 \\
\hline faces & 189,351 & 28,155 \\
\hline edges & 378,862 & 56,924 \\
\hline packfacetedge & 568,053 & 84,465 \\
\hline$\eta_{\text {mean }}$ & 1.54 & 1.48 \\
\hline history size & $4,106 \mathrm{~KB}$ & $625 \mathrm{~KB}$ \\
\hline HyT size & $10,765 \mathrm{~KB}$ & $1,622 \mathrm{~KB}$ \\
\hline HyT / history & 2.6 & 2.59 \\
\hline
\end{tabular}

Table 2: Memory size of the history and the hypertriangulation formats.

The overall complexity is therefore $O(n \log n)$, where $n$ is the number of faces produced/updated in each selective refinement.

Empirical time complexity depends on a number of factors: the size of the multiresolution model, the value of the face adjacency factor, the current focus point position, the radius length, and the current error function $E$. The empirical complexity was measured on a number of meshes using an SGI Indigo2 XZ (200MHz R4400 CPU, 16KB data and instruction cache, 1MB secondary cache). We obtained:

- constant precision extraction from the HyT scheme at a rate of approximately $110 \mathrm{~K}$ faces per second;

- selective refinements at a rate of approximately $15 \mathrm{~K}$ faces per second, with a mean size of 200 1000 faces extracted for each selective refinement action (i.e. an ideal rate higher than 15 frames per second; we call it ideal because user can't sustain such rate in driving the GUI).

The complexity of the meshes used in the tests was in the range $50 \mathrm{~K}$ - $200 \mathrm{~K}$ faces (e.g. the Bunny multiresolution mesh in Figure 9 holds $189 \mathrm{~K}$ faces). Both the above rates were measured by not performing graphics output to the OpenInventor toolkit (which is used by the Zeta system to manage graphics output). Even if we include the overhead due to the visualization of intermediate results by the OpenInventor toolkit, the system response is still interactive, with approximately sub-second response time. Being our goal the management of a user-driven modeling session, processing times are sufficiently fast.

The comparison of our performances with those of other multiresolution approaches is not simple. Extraction rates of 3,600 faces in $90 \mathrm{msec}$ were reported for the merge tree data structure [31], on the Bunny dataset and using a workstation slightly faster than ours (SGI R4400 at 250MHz). The extraction rate is in this case approximately $40 \mathrm{~K}$ faces per second.

The efficiency of view-dependent selective refinement using the progressive meshes structure is really impressive: according the the results reported by Hoppe [20], view-dependent meshes composed by $10 \mathrm{~K}$ triangles are extracted and rendered at approximately 7 frames per second (SGI Extreme R4400 150 MHz times). But here the goal is the dynamic, automatic generation of view dependent meshes, and a number of pre-computed measures are provided to speedup the run-time selection of the vertices to be refined/collapsed.

In the case of the Interactive Multiresolution Mesh Editing system [32], multiresolution rendering is operated in real time (the quadtree forest is visited adaptively, by taking into account the available rendering performance). The multiresolution editing actions are performed by moving sets of control points; small sets of points (which defines 20-30 faces at level 0 of the hierarchical representation, i.e. the coarser one) are interactively edited at a rate of 5 frames per second, on a SGI R10000 Solid Impact.

To evaluate different multiresolution modeling approaches, a first step is to validate if the interactivity 
issue is fulfilled (all of the above approaches, and ours as well, perform mesh editing or variable resolution extraction at interactive frame rates). Then, the evaluation has to be refined by comparing the respective quality of the results produced, because a comparison which takes into account only the extraction speed has a limited validity. This second evaluation stage is extremely difficult to be done theoretically, and an user-based evaluation is strictly needed. Moreover, the previous approaches are characterized by different objectives (view-dependent variable resolution mesh [31,20], shape modeling under a multiresolution representation [32], and direct modeling of resolution).

\section{Conclusions}

In this paper we presented a prototypal system which gives a unified solution to both the selective refinement problem and its reverse, selective simplification. The solution is designed according to a precise constraint: selective updates (either refinement or simplification) have to be operated incrementally over a variable resolution mesh. Moreover, the proposed approach must show an interactive response time. This technology, together with the large number of mesh simplification methods and multiresolution representation schemes recently developed, is the bases for a new modeling framework based on three separate stages (shape modeling, multiresolution encoding and resolution modeling). The purpose of resolution modeling is not limited to visualization speedup, but its main goal is to support interactive modeling of details. Possible applications have been sketched out, and we plan to further investigate on that.

Our approach to resolution modeling is highly general and user-driven. It can operate on a historybased multiresolution representation which can be produced with any iterative simplification approach based on local simplification and global error evaluation.

The approach proposed is based on a representation scheme, the Hypertriangulation (HyT), and a set of kernel functionalities which operate on the HyT to support: efficient extraction of fixed resolution representation; unified management of selective refinement and selective simplification; easy composition of the selective refinement/simplification actions; guaranteed $\mathcal{C}_{0}$ continuity of the variable resolution mesh produced; interactive response times.

Unlike other approaches based on the selective inversion of simplification actions (vertex decimation or edge collapsing), our proposal is based on a complete multiresolution encoding, and selective refinements/simplifications are operated through a topology-based navigation of the multiresolution representation. Multiresolution encoding resulted in limited memory overhead. On the other hand, it ensures high efficiency and flexibility in performing resolution modeling actions and easy incremental composition of the updates. Moreover, it can be paired with more sophisticated simplification methods.

Conversely to other approaches (e.g. multiresolution editing [32]), Zeta allows to edit the mesh at whichever level of detail, both to apply selective simplification/refinement actions or to perform shape editing, and it does not require the input mesh to possess subdivision connectivity (i.e. its applicability is wider).

\section{Acknowledgements}

This work was partially financed by the Progetto Coordinato "Modelli multirisoluzione per la visualizzazione di campi scalari multidimensionali" of the Italian National Research Council (C.N.R.). We wish also to thank Enrico Puppo of I.M.A.- C.N.R. and Leila De Floriani of the University of Genova for many 
useful discussions and comments.

\section{References}

[1] A. Certain, J. Popovic, T. DeRose, T. Duchamp, D. Salesin, and W. Stuetzle. Interactive multiresolution surface viewing. In Comp. Graph. Proc., Annual Conf. Series (Siggraph '96), ACM Press, pages 91-98, Aug. 6-8 1996.

[2] A. Ciampalini, P. Cignoni, C. Montani, and R. Scopigno. Multiresolution decimation based on global error. The Visual Computer, 13(5):228-246, June 1997.

[3] P. Cignoni, C. Montani, E. Puppo, and R. Scopigno. Multiresolution modeling and visualization of volume data. IEEE Trans. on Visualization and Comp. Graph., 3(4):352-369, 1997.

[4] P. Cignoni, E. Puppo, and R. Scopigno. Representation and visualization of terrain surfaces at variable resolution. The Visual Computer, 13(5):199-217, 1997.

[5] J. H. Clark. Hierarchical geometric models for visible surface algorithms. Comm. of the ACM, 19(10):547-554, Oct. 1976.

[6] J. Cohen, A. Varshney, D. Manocha, G. Turk, H. Weber, P. Agarwal, F. Brooks, and W. Wright. Simplification envelopes. In Computer Graphics Proc., Annual Conf. Series (Siggraph '96), ACM Press, pages 119-128, Aug. 6-8 1996.

[7] M. de Berg and K. Dobrindt. On levels of detail in terrains. In Proceedings 11th ACM Symposium on Computational Geometry, pages C26-C27, Vancouver (Canada), 1995. ACM Press.

[8] L. De Floriani, P. Marzano, and E. Puppo. Multiresolution models for topographic surface description. The Visual Computer, 12(7):317-345, 1996.

[9] D.P. Dobkin and M.J. Laszlo. Primitives for the manipulation of three-dimensional subdivisions. Algorithmica, 4:3-32, 1989.

[10] M.A. Duchaineau, M. Wolinsky, D.E. Sigeti, M.C. Miller, C. Aldrich, and M.B. Mineev-Weinstein. ROAMing terrain: Real-time optimally adapting meshes. In IEEE Visualization '97, October 1997.

[11] M. Eck, T. De Rose, T. Duchamp, H. Hoppe, M. Lounsbery, and W. Stuetzle. Multiresolution analysis of arbitrary meshes. In Computer Graphics Proc., Annual Conf. Series (Siggraph '95), ACM Press, pages 173-181, Aug. 6-12 1995.

[12] D.R. Forsey and R.H. Bartels. Hierarchical b-spline refinement. Computer Graphics (SIGGRAPH '88 Proceedings), 22(4):205-212, August 1988.

[13] R.J. Fowler and J.J. Little. Automatic extraction of irregular network digital terrain models. $A C M$ Computer Graphics (Siggraph '79 Proc.), 13(3):199-207, Aug. 1979.

[14] T.A. Funkhouser and C.H. Sequin. Adaptive display algorithm for interactive frame rates during visualization of complex environment. In Computer Graphics Proc., Annual Conf. Series (SIGGRAPH 93), pages 247-254. ACM Press, 1993. 
[15] G. Gallo and S. Pallottino. Shortest path algorithms. Annals of Operations Research, 13:3-79, 1988.

[16] M. Garland and P.S. Heckbert. Surface simplification using quadric error metrics. In Comp. Graph. Proc., Annual Conf. Series (Siggraph '97), ACM Press, pages 209-216, 1997.

[17] M.H. Gross, O.G. Staadt, and R. Gatti. Efficient triangular surface approximations using wavelets and quadtree data structures. IEEE Trans. on Visual. and Comp. Graph., 2(2):130-144, June 1996.

[18] P. Heckbert and M. Garland. Multiresolution Modeling for Fast Rendering. In Graphics Interface '94 Proceedings, pages 43-50, 1994.

[19] H. Hoppe. Progressive meshes. In ACM Computer Graphics Proc., Annual Conference Series, (Siggraph '96), pages 99-108, 1996.

[20] Hugues Hoppe. View-dependent refinement of progressive meshes. In ACM Computer Graphics Proc., Annual Conference Series, (Siggraph '97), 1997. 189-198.

[21] Hugues Hoppe, Tony DeRose, Tom Duchamp, John McDonald, and Werner Stuetzle. Mesh optimization. In ACM Computer Graphics Proc., Annual Conference Series, (Siggraph '93), pages 19-26, 1993.

[22] R. Klein, G. Liebich, and W. Straßer. Mesh reduction with error control. In R. Yagel and G. Nielson, editors, Proceedings of Visualization '96, pages 311-318, 1996.

[23] P. Lindstrom, D. Koller, W. Ribarsky, L.F. Hodges, N. Faust, and G.A. Turner. Real-time, continuous level of detail rendering of height fields. In Comp. Graph. Proc., Annual Conf. Series (Siggraph '96), ACM Press, pages 109-118, New Orleans, LA, USA, Aug. 6-8 1996.

[24] E. Puppo and R. Scopigno. Simplification, LOD, and Multiresolution - Principles and Applications. In EUROGRAPHICS'97 Tutorial Notes (ISSN 1017-4656). Eurographics Association, Aire-la-Ville (CH), 1997 (PS97 TN4).

[25] R. Ronfard and J. Rossignac. Full-range approximation of triangulated polyhedra. Computer Graphics Forum (Eurographics'96 Proc.), 15(3):67-76, 1996.

[26] J. Rossignac and P. Borrel. Multi-resolution 3D approximation for rendering complex scenes. In B. Falcidieno and T.L. Kunii, editors, Geometric Modeling in Computer Graphics, pages 455-465. Springer Verlag, 1993.

[27] Lori Scarlatos and Theo Pavlidis. Hierarchical triangulation using cartographics coherence. CVGIP: Graphical Models and Image Processing, 54(2):147-161, March 1992.

[28] William J. Schroeder, Jonathan A. Zarge, and William E. Lorensen. Decimation of triangle meshes. In Edwin E. Catmull, editor, ACM Computer Graphics (SIGGRAPH '92 Proceedings), volume 26, pages $65-70$, July 1992 .

[29] Greg Turk. Re-tiling polygonal surfaces. In Edwin E. Catmull, editor, ACM Computer Graphics (SIGGRAPH '92 Proceedings), volume 26, pages 55-64, July 1992. 
[30] J. Wilhelms and A. van Gelder. Multi-dimensional Trees for Controlled Volume Rendering and Compression. In Proceedings of 1994 Symposium on Volume Visualization, pages 27-34. ACM Press, October 17-18 1994.

[31] J.C. Xia, J. El-Sana, and A. Varshney. Adaptive real-time level-of-detail-based rendering for polygonal models. IEEE Trans. on Visualization and Computer Graphics, 3(2):171-183, 1997.

[32] D. Zorin, P. Schröder, and W. Sweldens. Interactive multiresolution mesh editing. In Comp. Graph. Proc., Annual Conf. Series (Siggraph '97), ACM Press, 1997. 259-268. 
Fixed error section

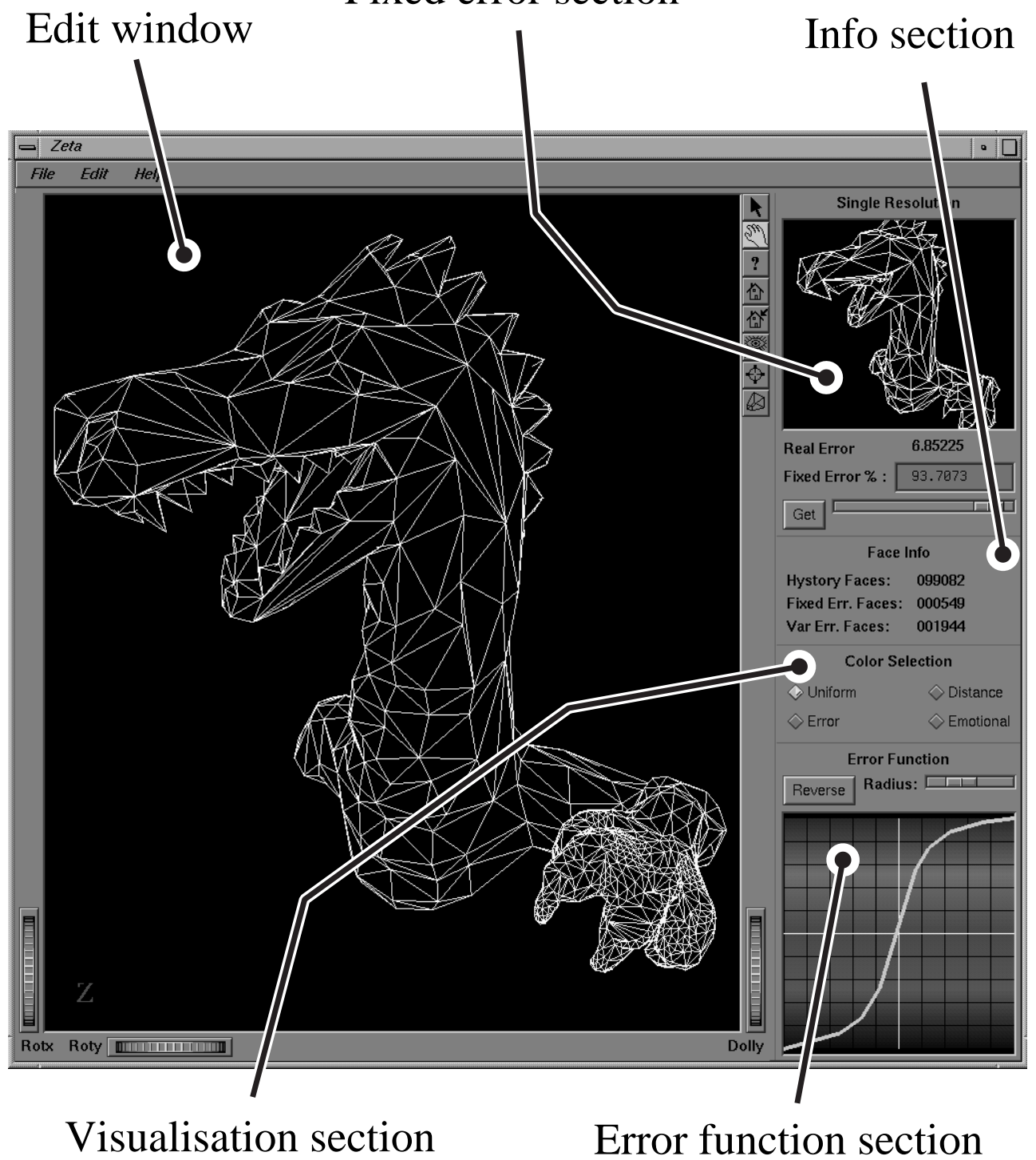

Figure 8: The interface of Zeta, our resolution modeling prototypal system. 

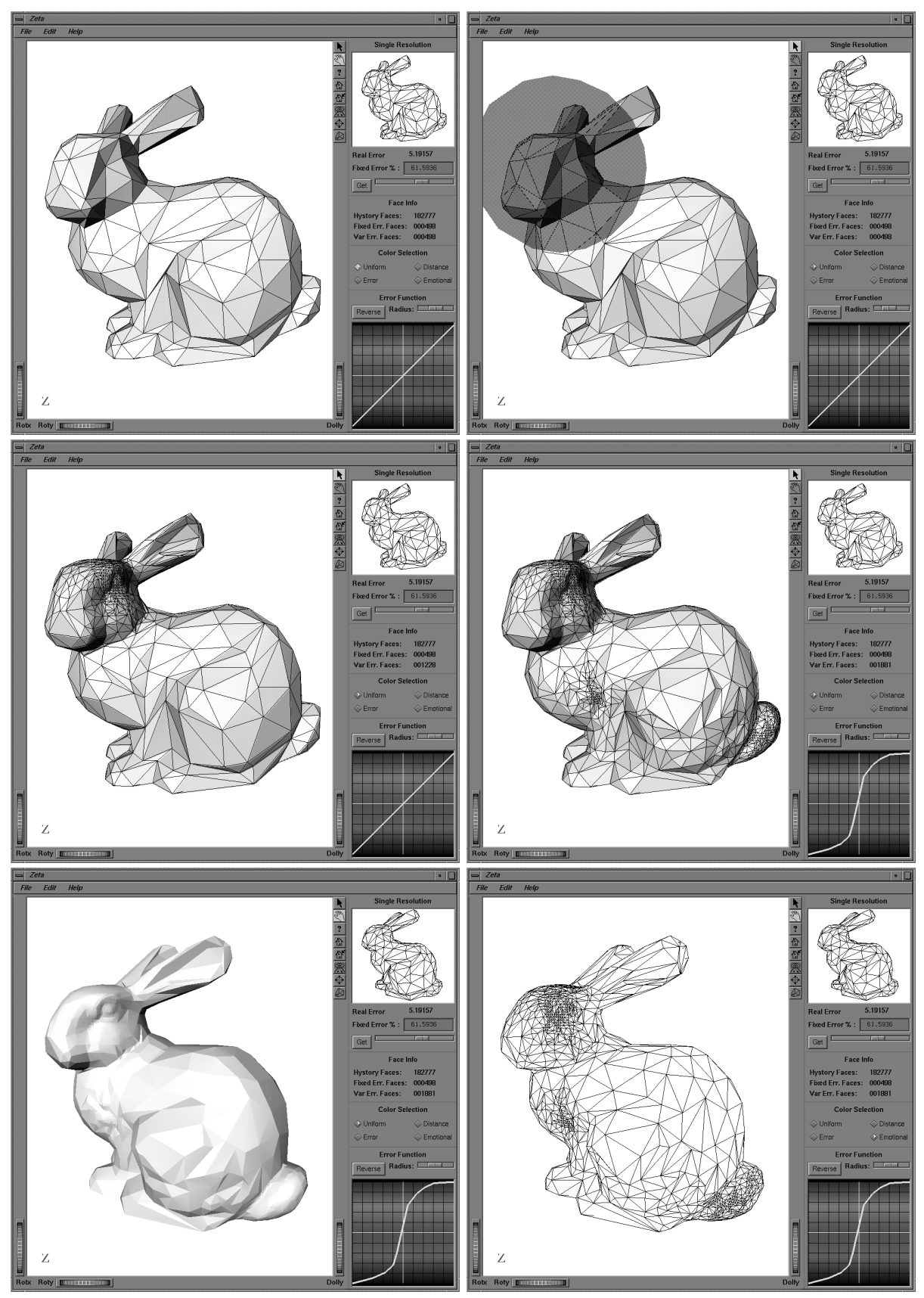

Figure 9: Some subsequent stages of a resolution modeling session (top-down, left-to-right): (a) selection of the initial fixed error mesh; (b) selection of the radius used (c) in the following selective refinement on the Bunny eye; (d) the error function $E$ has been modified and two more selective refinements have been operated on the cheek and the tail; (e,f) shaded and wire frame rendering of the final variable resolution mesh. 

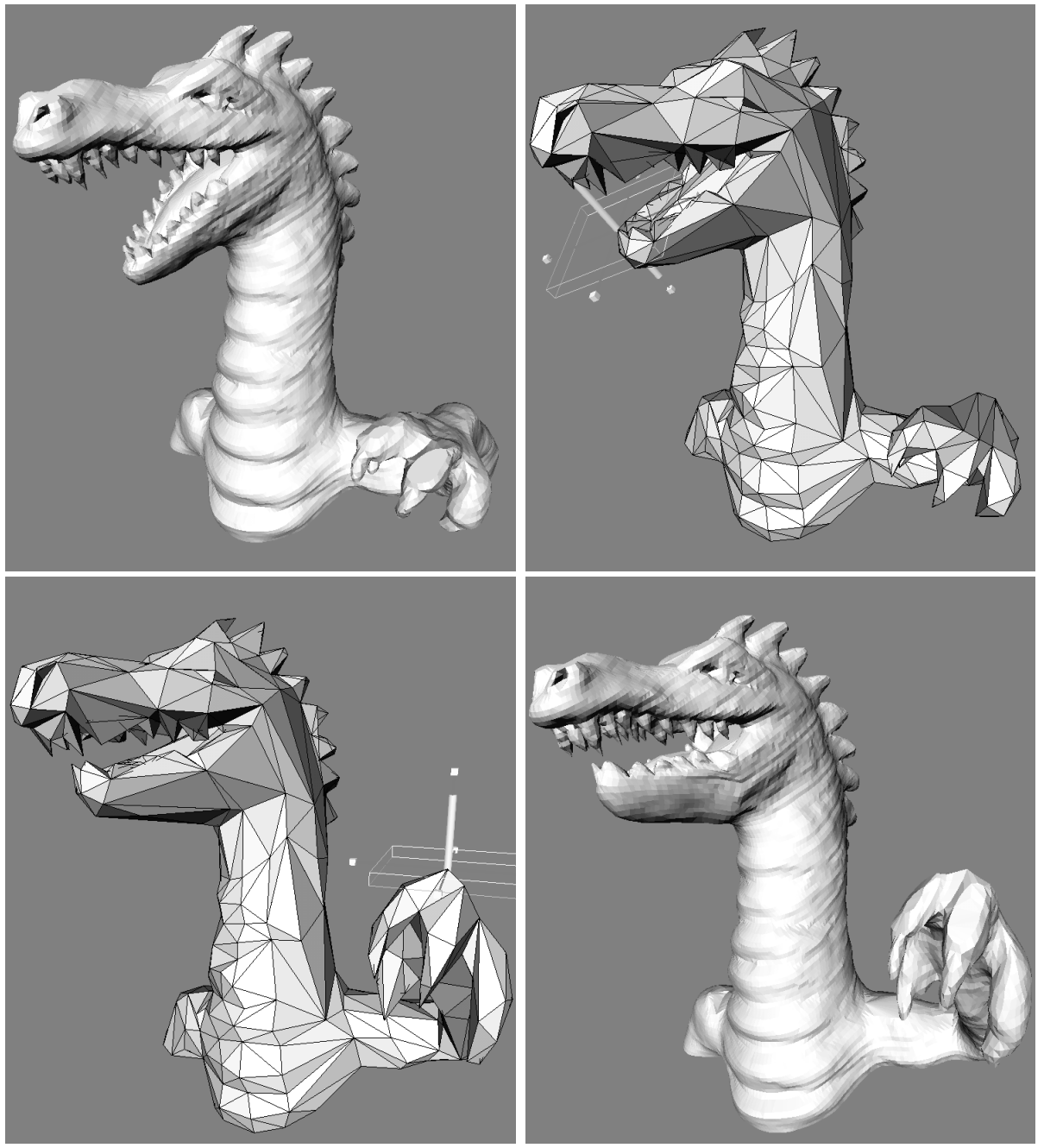

Figure 10: Some steps of an editing session. The initial mesh (at high resolution) is shown in the top-left image; then, a lower resolution was selected to perform shape editing: the crest was raised, and the user is now editing the jaw (top-right image); user is raising the dragon's hand (bottom-left image); the resulting edited mesh at high resolution (bottom-right image). 

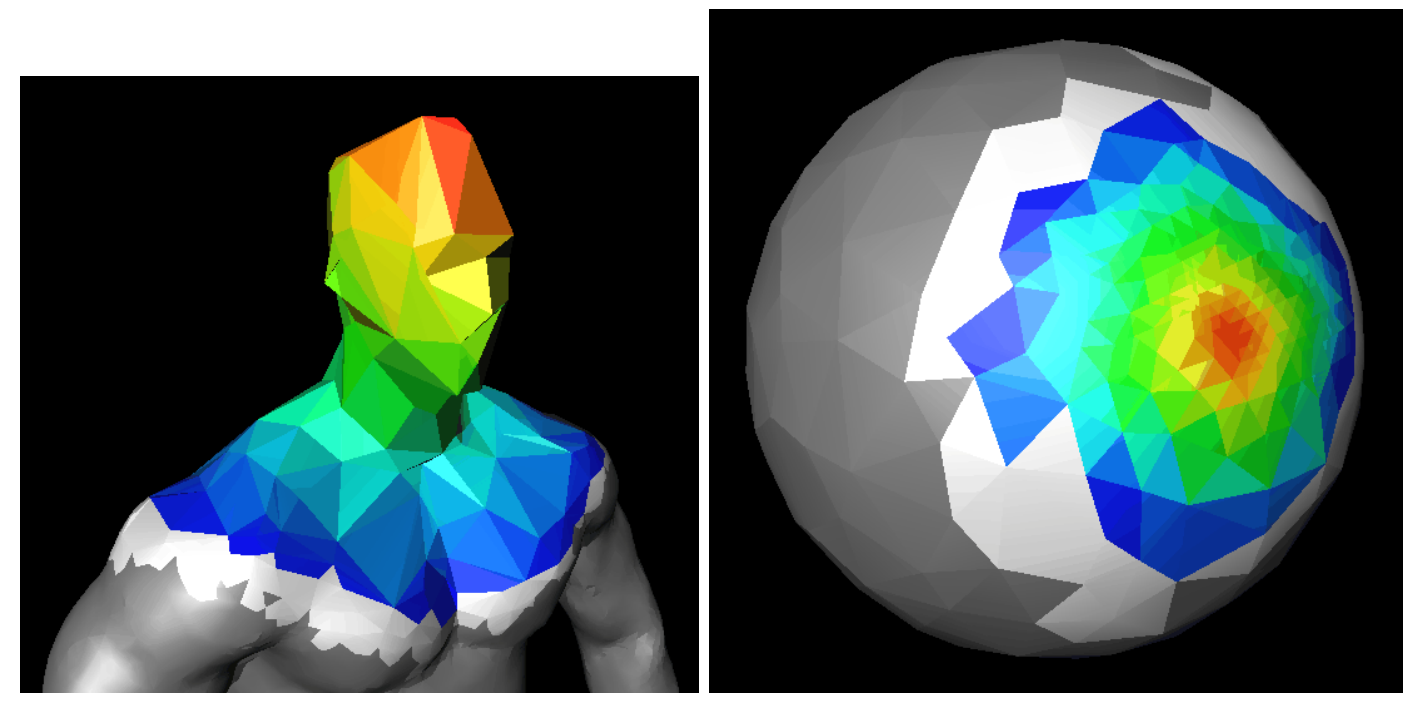

Figure 11: The updated mesh section, after a selective simplification (on the left) and a selective refinement (on the right); a color ramp (red-to-blue) represents the distance from the focus point, and the white faces are those produced at distance higher than the current radius.

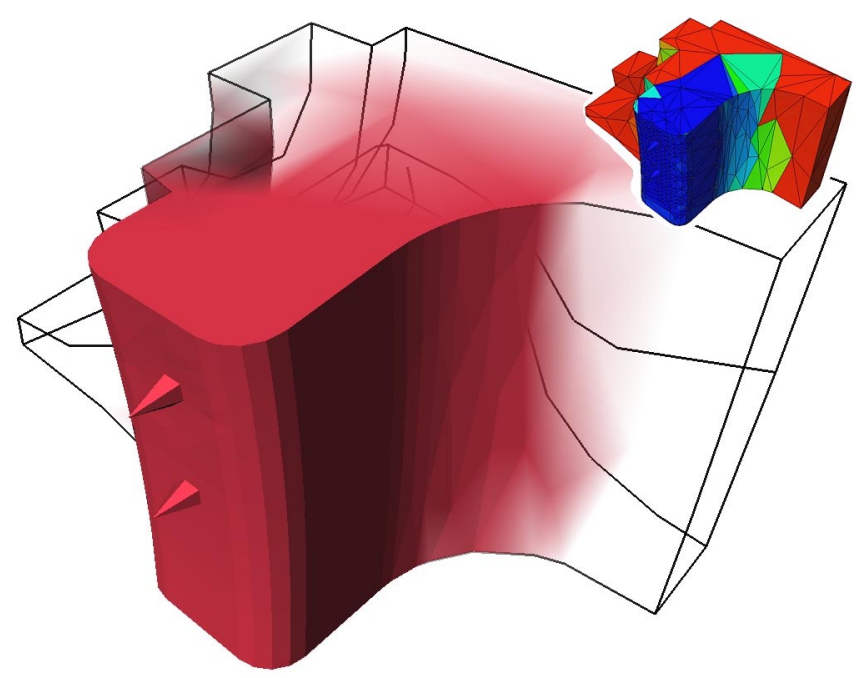

Figure 12: An example of the intermixing different rendering modes to enhance of informational content: only part of a gas turbine engine component ("fandisk") is well detailed while the rest of the mesh is just (literally) sketched by drawing the sharp edges only. 


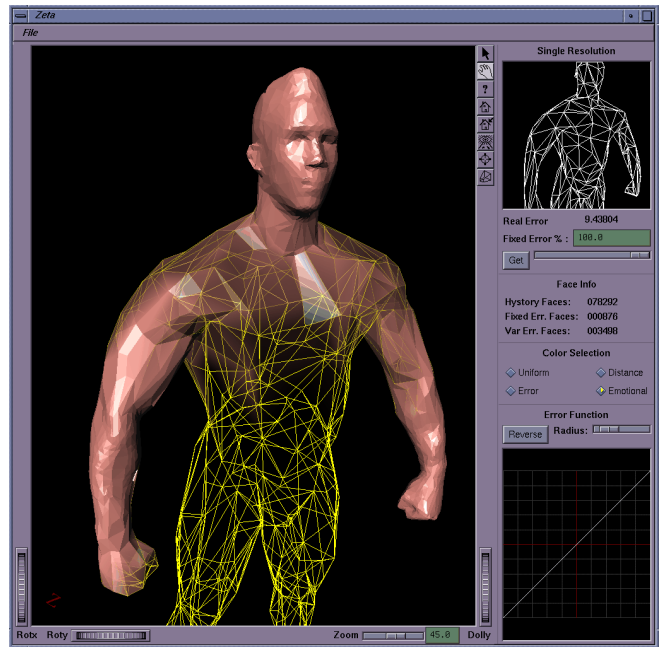

Figure 13: Another example of the use of two rendering modes; few composed refinement actions were operated in the areas of the head and the arms, and also these high resolution sections are rendered under shaded mode. 